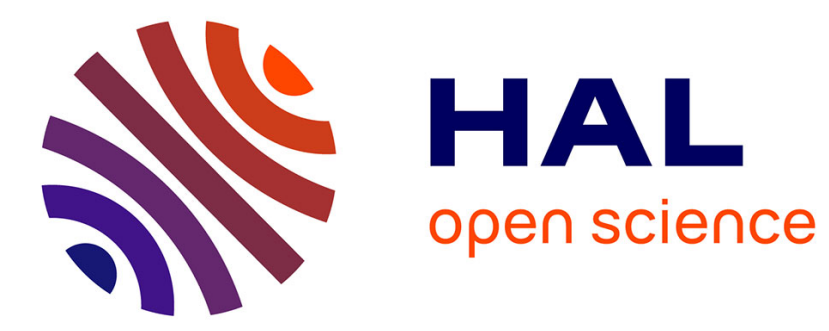

\title{
Spectromètre à paramètres optiques réglables
}

\author{
C. Bazin, J. Dupin
}

\section{To cite this version:}

C. Bazin, J. Dupin. Spectromètre à paramètres optiques réglables. Revue de Physique Appliquée, 1969, 4 (2), pp.201-202. 10.1051/rphysap:0196900402020100 . jpa-00243218

\section{HAL Id: jpa-00243218 https://hal.science/jpa-00243218}

Submitted on 1 Jan 1969

HAL is a multi-disciplinary open access archive for the deposit and dissemination of scientific research documents, whether they are published or not. The documents may come from teaching and research institutions in France or abroad, or from public or private research centers.
L'archive ouverte pluridisciplinaire HAL, est destinée au dépôt et à la diffusion de documents scientifiques de niveau recherche, publiés ou non, émanant des établissements d'enseignement et de recherche français ou étrangers, des laboratoires publics ou privés. 


\title{
SPECTROMÈTRE A PARAMÈTRES OPTIQUES RÉGLABLES
}

\author{
G. BAZIN et J. DUPIN, \\ École Normale Supérieure, Laboratoire de l'Accélérateur Linéaire, 91-Orsay.
}

\begin{abstract}
Résumé. - La présence de nappes polaires de courant assure à ce spectromètre des propriétés optiques constantes en fonction de l'induction. Ces propriétés concernent les coefficients du premier et du second ordre, l'angle de la droite focale et la dispersion. On a montré ainsi que le domaine d'utilisation à caractéristiques optiques constantes de cet appareil pouvait être étendu de 0,8 à $1,27 \mathrm{GeV} / \mathrm{c}$.
\end{abstract}

Abstract. - A system of pole-face windings provides this spectrometer with magnetic characteristics independent of the induction. These characteristics concern the first and second order coefficients, the focal plane angle and the dispersion. We, thus, demonstrate that the range over which the optical characteristics are constant have, by this method, been extended from 0.8 to $1.27 \mathrm{GeV} / \mathrm{c}$.

Le spectromètre $1,3 \mathrm{GeV} / \mathrm{c}$ est un électroaimant à double focalisation prévu pour être utilisé en arrangement symétrique. Ses principales caractéristiques mécaniques sont : i) angle de déflexion $\theta=180^{\circ}$; ii) rayon de courbure $R_{0}=2,17 \mathrm{~m}$; iii) entrefer à $R_{0}$, $e=0,27 \mathrm{~m}$. Sa particularité est de posséder des nappes polaires de conducteurs qui lui permettent d'obtenir des propriétés optiques indépendantes de l'induction.

Nous donnons, ci-dessous, la variation des principaux paramètres lorsque la quantité du mouvement $p$ des particules analysées varie de 1,3 à $0,1 \mathrm{GeV} / \mathrm{c}$ (sans utilisation des nappes polaires) :

- indice ou coefficient du 1 er ordre, $0,36 \leqslant n \leqslant 0,50$;

- coefficient du second ordre, $-1,30 \leqslant \beta \leqslant 0,35$;

- angle de la droite focale, $9^{\circ} \leqslant F \leqslant 58^{\circ}$;

- dispersion, $8,1 \leqslant D \leqslant 8,6 \mathrm{~cm} / \%$;

- angle solide, $4,4 \leqslant \frac{\Omega}{10^{-3}} \leqslant 7$ sr.

La détermination expérimentale des coefficients du premier et du second ordre s'est faite à partir d'une méthode simple [1] : déplacement à vitesse constante d'une bobine de surface équivalente connue dans un champ magnétique où seule la valeur de l'induction à $R_{0}$ est nécessaire. La tension délivrée par la bobine, après amplification, est affichée sur un voltmètre digital, enregistrée et imprimée. La mesure est faite pour des points équidistants, le déclenchement se faisant par microswitch à commande magnétique. Pour l'angle de la focale et la dispersion, la méthode du fil flottant utilisant une poulie-balance [2] a été employée. L'avantage de ce système réside dans la possibilité de fixer le point d'accrochage du fil au-delà du point image, donc de déterminer les rayons dans l'espace image avec une plus grande précision.

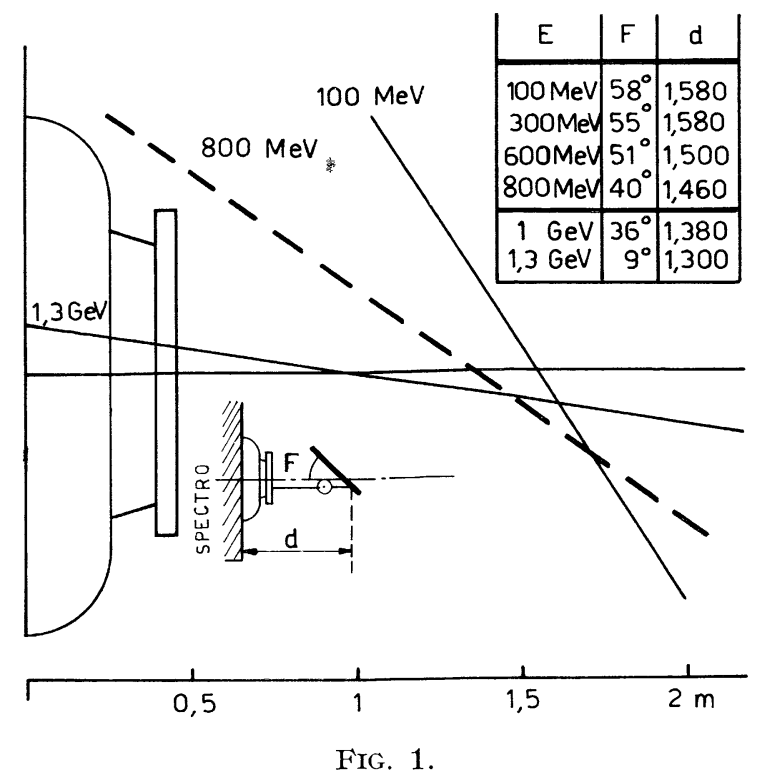

Variation de l'angle de la focale en fonction de l'énergie.

A cause des variations aussi importantes que celles notées précédemment, la double focalisation n'est obtenue que pour une valeur de la quantité de mouvement voisine de $1 \mathrm{GeV} / \mathrm{c}$. Les aberrations étant alors minimales, nous avons aussi une meilleure résolution $\left(2 \times 10^{-4}\right.$ pour $\left.\Omega=2 \times 10^{-3} \mathrm{sr}\right)$.

Nappes correctrices. - Le principe des nappes est celui des circuits correcteurs du synchrotron Saturne [3]. Les coefficients du premier et du second ordre sont corrigés chacun par une nappe dont les caractéristiques 


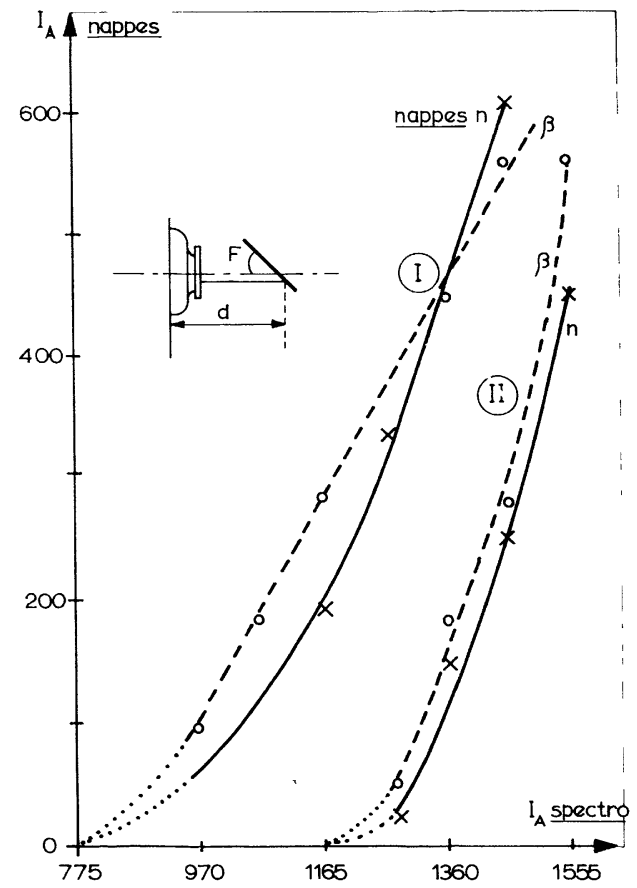

FIG. 2. - Courant dans les nappes $n$ et $\beta$ en fonction du courant dans le spectromètre pour les angles de valeurs : I : $F=40^{\circ} ; d=1,46 \mathrm{~m}$ et II : $F: 24^{\circ}$; $d=1,06 \mathrm{~m}$. sont différentes. Sur chaque pôle on compte 15 conducteurs équidistants (nappe $n$ ), 12 conducteurs groupés par 3 (nappe $\beta$ ). Les conducteurs sont des tubes de cuivre de $\varnothing$ extérieur $10 \mathrm{~mm}, \varnothing$ intérieur $8 \mathrm{~mm}$, maintenus et isolés du pôle par des barrettes de silirite (matière isolante amagnétique et ayant une bonne tenue aux radiations). Une étude préliminaire [4] sur une maquette à l'échelle $1 / 6$ a montré que la correction apportée par une nappe était pratiquement sans influence sur l'autre nappe.

La présence des nappes polaires correctrices d'indice nous a permis d'obtenir une double focalisation pour des particules dont la quantité de mouvement varie de 0,8 à $1,27 \mathrm{GeV} / \mathrm{c}$, et une droite focale immobile avec une valeur de l'angle $F$ ajustable :

$$
F=40^{\circ} ; \quad d=1,46 \mathrm{~m}
$$

pour $0,8 \leqslant p \leqslant 1,27 \mathrm{GeV} / \mathrm{c}$

$$
F=24^{\circ} ; \quad d=1,06 \mathrm{~m}
$$

pour $1,17 \leqslant p \leqslant 1,3 \mathrm{GeV} / \mathrm{c}$.

Enfin, une dispersion à $1,3 \mathrm{GeV} / \mathrm{c}$ de $D=8,4 \mathrm{~cm} / \%$.

De plus, la constance des coefficients du premier et du second ordre entraîne pour les aberrations angulaires et chromatiques des taches de dimensions plus petites. Ainsi, à $1,2 \mathrm{GeV} / \mathrm{c}$, alors que la dimension de l'aberration était de $10 \mathrm{~mm}$, elle se trouve réduite à $6 \mathrm{~mm}$ lorsqu'on utilise les nappes.

\section{BIBLIOGRAPHIE}

[1] C'AMPlan (J.) et Meunier (R.), Nucl. Instr. Methods, 1964, 31, 92.

[2] Bounin (P.) et Mirman (B.), Rev. Sci. Instr., 1963, $34,12$.
[3] NeYret (G.) et Parain (J.), Nucl. Instr. Methods, 1959, 5, 259.

[4] Boutourye (B.) et Fardeau (P.), J. Phys. Appl., 1965, 26, 27 A. 